\title{
Influence of a Headrest on Reconstruction and Attenuation Correction of Human Brain SPECT Images
}

\author{
Makoto Ohba ${ }^{1}$, Yasuaki Kokubo ${ }^{2}$, Koji Suzuki ${ }^{1}$, Masafumi Kanoto ${ }^{3}$, and Yukihiko Sonoda ${ }^{2}$ \\ ${ }^{1}$ Department of Radiology, Yamagata University Hospital, Yamagata, Japan; ${ }^{2}$ Department of Neurosurgery, Faculty of Medicine, \\ Yamagata University, Yamagata, Japan; and ${ }^{3}$ Division of Diagnostic Radiology, Department of Radiology, Yamagata University \\ Graduate School of Medical Science, Yamagata, Japan
}

\begin{abstract}
Previous reports suggest that a headrest significantly influences anterior and posterior cerebral blood flow. The present study aimed to clarify the influence of a headrest on reconstruction and attenuation correction (AC) of brain SPECT images. Methods: We evaluated the influence on cerebral blood flow in the anterior region (brain segments $A+B$ ), middle region (segments $D+F$ ), and posterior region (segment $\mathrm{G}$ ) of the brain using filtered backprojection-AC based on the method of Chang (FBP-ChangAC), ordered-subset expectation maximization-ChangAC (OSEMChangAC), OSEM CT-based AC (OSEM-CTAC), and OSEM with no attenuation correction (OSEM-NoAC) with and without a headrest. The subjects were 17 healthy volunteers who underwent ${ }^{99 m}$ Tc-ECD SPECT. We compared the $A+B / G$ and the $D$ $+F / G$ ratios of $99 m T c-E C D$ SPECT images in each group. $\mathbf{R e}-$ sults: For FBP-ChangAC, OSEM-ChangAC, and OSEM-NoAC, there were significant differences in $A+B / G$ ratio between images obtained with a headrest and those obtained without. On the other hand, for OSEM-CTAC, there were no significant differences in $A+B / G$ ratio regardless of whether a headrest was used. For FBP-ChangAC and OSEM-NoAC, there were significant differences in $D+F / G$ ratio between images with a headrest and those without. For OSEM-CTAC and OSEM-ChangAC, there were no significant differences in $D+F / G$ ratio regardless of whether a headrest was used. Conclusion: The influence of a headrest on image reconstruction and $A C$ should be considered if FBP-ChangAC, OSEM-ChangAC, or OSEM-NoAC is used but not if OSEM-CTAC is used.
\end{abstract}

Key Words: SPECT; headrest; image reconstruction; CT-based attenuation correction; ChangAC

J Nucl Med Technol 2021; 49:54-57

DOI: 10.2967/jnmt.120.244731

\section{I} maging of cerebral blood flow through SPECT plays an important role in the diagnosis of not only ischemic cerebrovascular disease but also dementia, other degenerative diseases, and psychiatric disorders. A statistical imaging analysis using

\footnotetext{
Received Mar. 9, 2020; revision accepted Jul. 25, 2020.

For correspondence or reprints contact: Yasuaki Kokubo, Yamagata University, 2-2-2 lida-Nishi, Yamagata, 990-9585 Japan.

E-mail: ykokubo@med.id.yamagata-u.ac.jp

Published online Sep. 4, 2020.

COPYRIGHT (C) 2021 by the Society of Nuclear Medicine and Molecular Imaging.
}

an easy $z$-score imaging system (e-ZIS) $(1,2)$ has become established as a useful tool for the differential diagnosis of conditions such as Alzheimer disease $(3,4)$ and Lewy body dementia $(5,6)$.

The possibility of interactions among various image reconstruction parameters should be considered, as such interactions may result in decreased image contrast and errors requiring compensation by attenuation correction (AC) and scatter correction. At present, there are $2 \mathrm{AC}$ methods for SPECT images: $\mathrm{AC}$ based on the method of Chang (ChangAC) (7) and $\mathrm{AC}$ based on CT (CTAC) $(8,9)$. ChangAC is based on the assumption of uniform attenuation of $\gamma$-rays by intracranial tissues. However, because of the presence of complex structures, such as air, soft tissues, the paranasal sinus, and various levels of skull thickness, accurate attenuation correction is difficult to achieve (10).

The recent growth of SPECT/CT has led to the application of CTAC, which is expected to be more accurate than ChangAC. Even for intracranial tissue that is not uniform with the CT-derived attenuation coefficient distribution, attenuation can be corrected by CTAC. Ishii et al. reported that frontal dominant hyperperfusion and parieto-occipital and cerebellar hypo-perfusion in brain SPECT images reconstructed with ChangAC compared to those reconstructed with AC-CT. Thus, compared with ChangAC, CTAC might reduce the influence of the headrest. However, at present, ChangAC is still frequently applied in the reconstruction of SPECT images because SPECT/CT is not widely available. Furthermore, it has been shown that a headrest can significantly influence cerebral blood flow in the anterior and posterior regions of the brain corrected by ChangAC (11).

To our knowledge, thus far no reports have evaluated the effect of any type of AC with and without a headrest on the evaluation of cerebral blood flow in the anterior and posterior regions of the brain. The present study aimed to clarify the influence of a headrest on reconstruction and $\mathrm{AC}$ of brain SPECT images.

\section{MATERIALS AND METHODS}

The study protocol was approved by the Ethical Review Committee of the Yamagata University Faculty of Medicine for epidemiologic research. All subjects gave written informed consent. 


\section{Subjects}

We enrolled 17 healthy male volunteers (mean age, $41.2 \pm 11.38$ $\mathrm{y}$; range, 26-59 y) who had no medical history of brain disease. They were assessed by brain ${ }^{99 m}$ Tc-ethyl cysteinate dimer (ECD) (12) SPECT with and without a headrest from July 2016 to March 2018.

\section{SPECT Acquisition}

Images were acquired by ${ }^{99 m}$ Tc-ECD SPECT/CT using a Symbia T2 with a rotating, dual-detector $\gamma$-camera and a low-energy, high-resolution collimator (Siemens Healthineers), with $360^{\circ}$ of continuous rotation during the acquisition $(2.5 \mathrm{~min} /$ rotation $\times 6$ rotations). For SPECT acquisitions without a headrest, the subjects were positioned laterally to make the patients more comfortable and reduce motion artifacts (Fig. 1). SPECT images were acquired for $15 \mathrm{~min}$, followed by a CT acquisition. The subjects were intravenously injected with $600 \mathrm{MBq}$ of ${ }^{99 \mathrm{~m} T c-E C D}$ while lying supine with their eyes closed and their head on a headrest. Magnification was $1.45 ; 128 \times 128$ matrices $(3.3 \mathrm{~mm} /$ pixel $)$ were used, the main window was $140 \pm 10.5 \mathrm{keV}$, and the subwindow was $7 \%$.

\section{Image Analysis}

Images were reconstructed by filtered backprojection (FBP) (13) and ordered-subset expectation maximization (OSEM) (14) with combined AC and scatter correction. The ChangAC used in FBP and OSEM creates an attenuation coefficient distribution that does not consider the headrest, as the correction applies only to intracranial tissue. The CTAC used in OSEM creates an attenuation coefficient distribution of CT data and corrects both the intracranial tissue and the headrest. Scatter was corrected in SPECT using a multienergy window.

We analyzed ${ }^{99 m} \mathrm{Tc}-\mathrm{ECD}$ SPECT images using a 3-dimensional stereotactic region-of-interest template (Fujifilm Toyama Chemical, Co., Ltd.) (15) that groups the regions into 12 segments (A, callosomarginal; B, precentral; C, central; D, parietal; E, angular; F, temporal; G, posterior; H, pericallosal; I, lentiform nuclear; J, thalamic; K, hippocampal; and L, cerebellar) in each hemisphere (Fig. 2). Focusing on the anterior region (segments $\mathrm{A}+\mathrm{B}$ ), the middle region (segments $\mathrm{D}$ $+F$ ), and the posterior region (segment $G$ ), we evaluated the influence of 4 reconstruction and correction methods (FBP-ChangAC, OSEMChangAC, OSEM-CTAC, and OSEM with no attenuation correction [OSEM-NoAC]) on A + B/G count ratio and $D+F / G$ count ratio. The results with and without a headrest were compared.

All statistical analyses were performed using the SPSS software program (version 26; SPSS Inc.). Differences in the A $+B / G$ and $\mathrm{D}+\mathrm{F} / \mathrm{G}$ ratios among the 4 methods with and without a headrest were analyzed by unpaired $t$ testing. $P$ values of less than 0.05 were considered statistically significant.

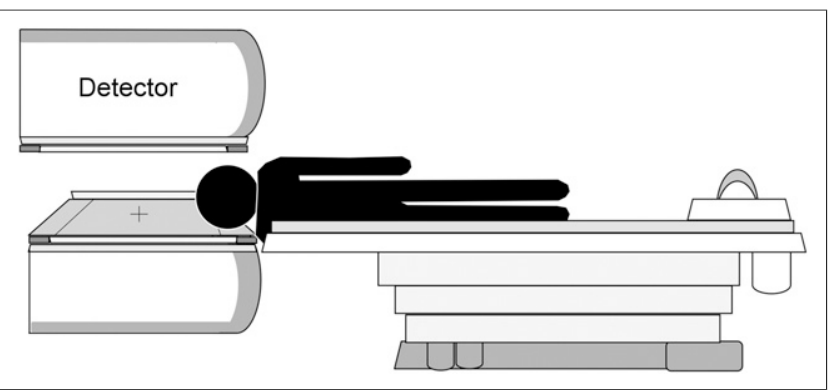

FIGURE 1. Subject positioned laterally to reduce discomfort and motion artifacts for SPECT acquisition without headrest.

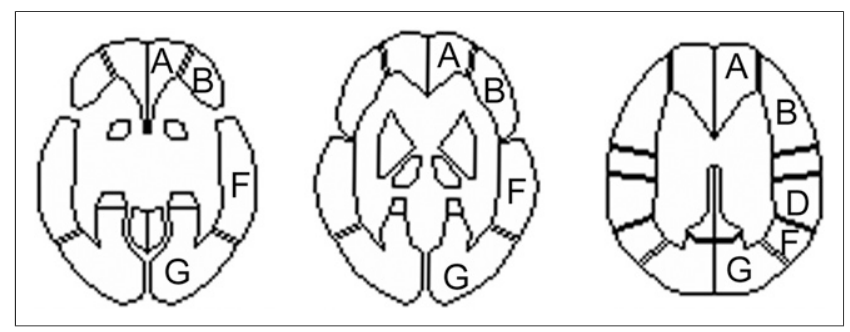

FIGURE 2. A 3-dimensional stereotactic region-of-interest template. Region $\mathrm{A}$ is callosomarginal territory, and region $\mathrm{B}$ is precentral territory; these two are defined as anterior brain region. Region $\mathrm{D}$ is parietal territory, and region $\mathrm{F}$ is temporal territory; these two are defined as middle brain region. Region $\mathrm{G}$ is posterior territory, which is defined as posterior brain region

\section{RESULTS}

Table 1 shows the average $A+B / G$ ratio for each group of images. There were significant differences in this ratio between images with a headrest and those without using FBPChangAC, OSEM-ChangAC, and OSEM-NoAC. On the other hand, there were no significant differences in this ratio, regardless of whether a headrest was used, for OSEM-CTAC. Table 2 shows the average $\mathrm{D}+\mathrm{F} / \mathrm{G}$ ratio for each group of images. There were significant differences in this ratio between images with a headrest and those without using FBPChangAC and OSEM-NoAC. On the other hand, there were no significant differences in this ratio, regardless of whether a headrest was used, for OSEM-CTAC and OSEM-ChangAC.

Figure 3 shows representative images at the level of the basal ganglia. For the anterior brain region $(\mathrm{A}+\mathrm{B})$, images using FBP-ChangAC, OSEM-ChangAC, and OSEM-NoAC with a headrest showed a higher count ratio than those without a headrest, and images using OSEM-CTAC with a headrest did not differ from those without a headrest. For the middle brain region $(\mathrm{F})$, images using FBP-ChangAC and OSEMNoAC with a headrest showed a higher count ratio than those without a headrest, and images using OSEM-ChangAC and OSEM-CTAC with a headrest did not differ from those without a headrest.

TABLE 1

Comparison of $A+B / G$ Ratio

\begin{tabular}{lccc}
\hline \multirow{2}{*}{$\begin{array}{c}\text { Reconstruction } \\
\text { method }\end{array}$} & \multicolumn{2}{c}{$\mathrm{A}+\mathrm{B} / \mathrm{G}$ ratio $(n=17)$} & \\
\cline { 2 - 3 } & $\begin{array}{c}\text { With } \\
\text { headrest }\end{array}$ & $\begin{array}{c}\text { Without } \\
\text { headrest }\end{array}$ & $P$ \\
\hline FBP-ChangAC & 0.97 & 0.91 & 0.008 \\
OSEM-CTAC & 0.91 & 0.92 & 0.35 \\
OSEM-ChangAC ${ }^{\dagger}$ & 0.98 & 0.92 & 0.03 \\
OSEM-NoAC ${ }^{*}$ & 1.04 & 0.98 & 0.002 \\
& & & \\
\hline${ }^{*} P<0.01$. & & & \\
${ }^{\dagger} P<0.05$. & & & \\
\hline
\end{tabular}


TABLE 2

Comparison of D + F/G Ratio

\begin{tabular}{lccc}
\hline \multirow{2}{*}{$\begin{array}{c}\text { Reconstruction } \\
\text { method }\end{array}$} & \multicolumn{2}{c}{$\mathrm{D}+\mathrm{F} / \mathrm{G}$ ratio $(n=17)$} & \\
\cline { 2 - 3 } & $\begin{array}{c}\text { With } \\
\text { headrest }\end{array}$ & $\begin{array}{c}\text { Without } \\
\text { headrest }\end{array}$ & $P$ \\
\hline FBP-ChangAC & 0.90 & 0.86 & 0.01 \\
OSEM-CTAC & 0.88 & 0.88 & 0.97 \\
OSEM-ChangAC & 0.90 & 0.87 & 0.06 \\
OSEM-NoAC ${ }^{\dagger}$ & 0.96 & 0.93 & 0.034 \\
& & & \\
\hline${ }^{*} P<0.01$. & & & \\
${ }^{\dagger} P<0.05$. & & & \\
\hline
\end{tabular}

Figure 4 shows representative images at the level of the ventricular body. For the anterior brain region $(\mathrm{A}+\mathrm{B})$, images using FBP-ChangAC, OSEM-ChangAC, and OSEM-NoAC with a headrest showed a higher count ratio than those without a headrest, and images using OSEM-CTAC with a headrest did not differ from those without a headrest. For the middle brain region $(\mathrm{D}+\mathrm{F})$, images using FBP-ChangAC and OSEM-NoAC with a headrest showed a higher count ratio than those without a headrest, and images using OSEM-ChangAC and OSEM-CTAC with a headrest did not differ from those without a headrest.

\section{DISCUSSION}

This study revealed significant differences in $A+B / G$ ratio for FBP-ChangAC, OSEM-ChangAC, and OSEMNoAC between images obtained with a headrest and those obtained without. In contrast, regardless of whether a headrest was used, there were significant differences in $\mathrm{D}+\mathrm{F} / \mathrm{G}$ ratio for FBP-ChangAC and OSEM-NoAC. Thus, the anterior brain region is more affected by a headrest in image reconstruction and AC. Ishii et al. reported that on SPECT images reconstructed using FBP and ChangAC, cerebral blood flow tended to be higher in the anterior brain than in the posterior brain (11), as is in line with our results. They suggested that a headrest made of carbon might influence attenuation of the posterior brain when SPECT images are reconstructed with FBP and ChangAC. Image reconstruction methods should consider the progression of $\gamma$-rays from the intracranial tissue through the headrest to the detector.

According to our results, the only reconstruction method that produced no significant difference in $A+B / G$ ratio, regardless of use of a headrest, was OSEM-CTAC. Therefore, SPECT images of the anterior brain with a headrest are more affected by ChangAC than by CTAC. The reason for this difference is that the CTAC used in OSEM corrects both the intracranial tissue and the headrest, whereas Chan$\mathrm{gAC}$, which is based on the assumption of uniform attenuation of $\gamma$-rays by intracranial tissues, does not consider the headrest-the correction applies only to intracranial tissue. Because the brain parenchyma and skull are structurally heterogeneous and attenuate $\gamma$-rays considerably, attenua- tion in intracranial tissue is unlikely to be uniform (16). One previous report indicated that the region contacting the headrest might also be overcorrected (11). Another previous report found that in SPECT reconstruction by OSEMCTAC, the influence of the headrest was minimal (10).

The principle of FBP is to obtain the SPECT image by back-projecting and filtering the projection data (13). It is well known that, compared with FBP, accuracy is higher for OSEM (14), which obtains the image by using a statistical method to estimate the image with the highest probability from the projection data, based on the radioactive distribution in the body. However, according to our results, OSEMChangAC is still affected by the presence of a headrest; if the

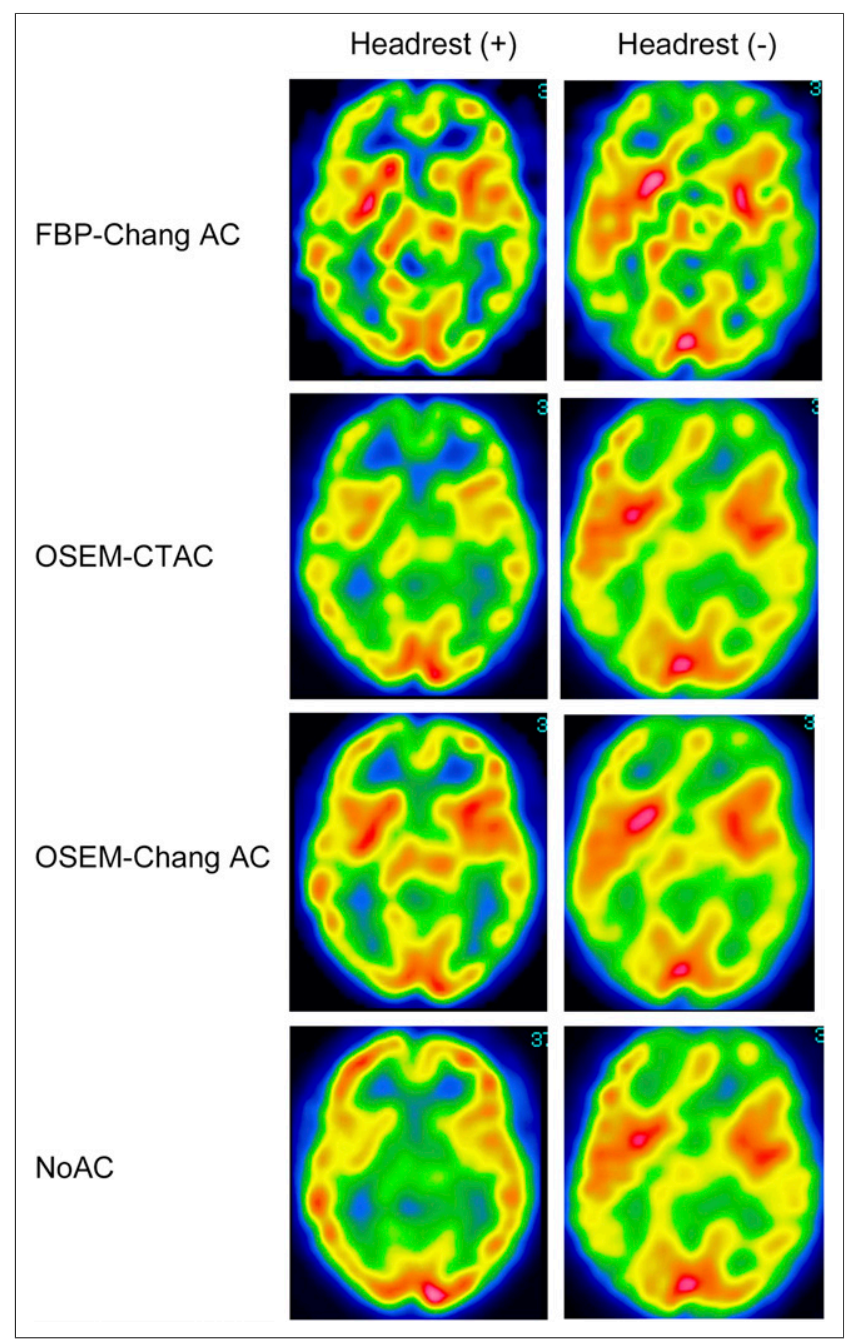

FIGURE 3. $\quad{ }^{99 m T C-E C D ~ S P E C T ~ i m a g e s ~ a t ~ l e v e l ~ o f ~ b a s a l ~ g a n g l i a . ~}$ Images of anterior brain region $(A+B)$ obtained using FBPChangAC, OSEM-ChangAC, and OSEM-NoAC with headrest were higher than those without headrest. Images of anterior brain region obtained using OSEM-CTAC with headrest did not differ from those without headrest. Images of middle brain region (F) obtained using FBP-ChangAC and OSEM-NoAC with headrest were higher than those without headrest. Images of middle brain region (F) obtained using OSEM-ChangAC and OSEM-CTAC with headrest did not differ from those without headrest. 


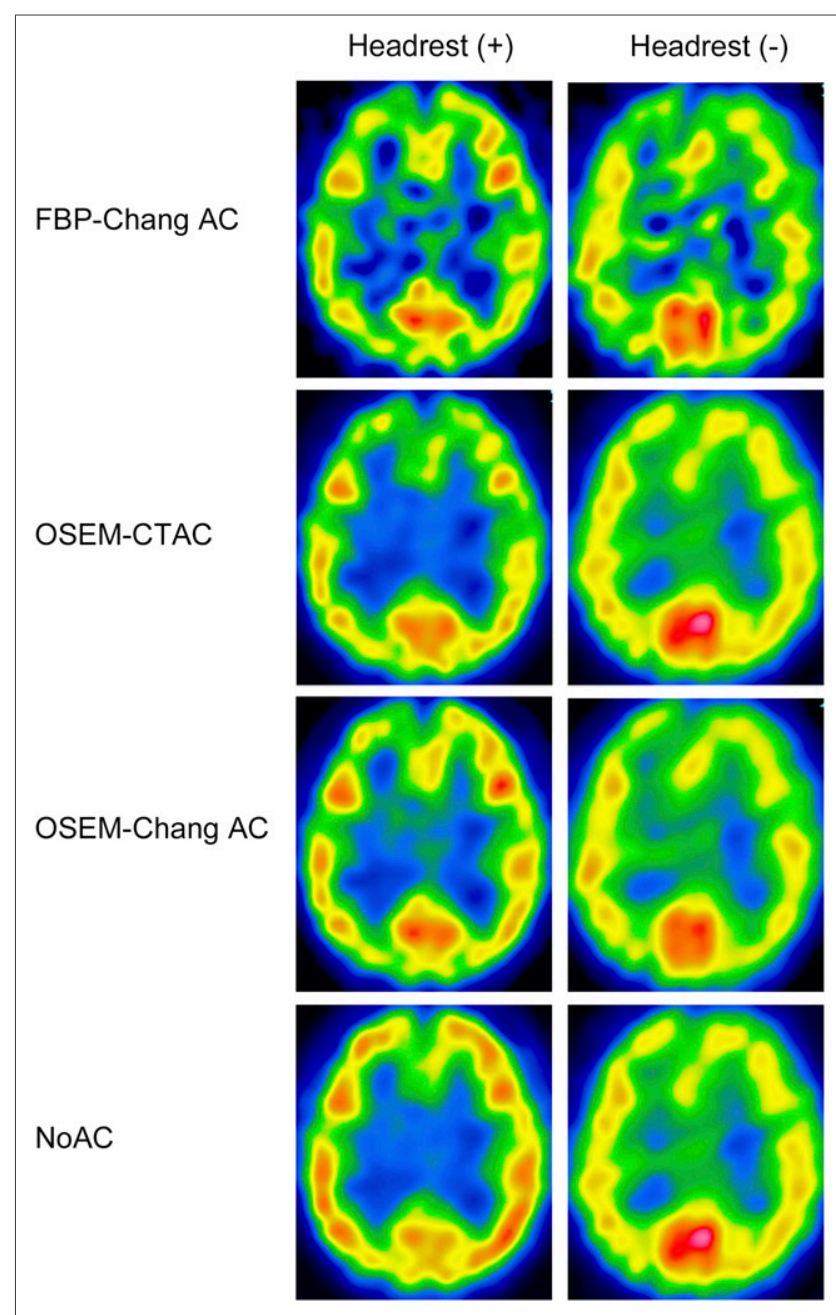

FIGURE 4. ${ }^{99 m T C-E C D ~ S P E C T ~ i m a g e s ~ a t ~ l e v e l ~ o f ~ v e n t r i c u l a r ~}$ body. Images of anterior brain region $(A+B)$ obtained using FBP-ChangAC, OSEM-ChangAC, and OSEM-NoAC with headrest were higher than those without headrest. Images of anterior brain region obtained using OSEM-CTAC with headrest did not differ from those without headrest. Images of middle brain region $(D+F)$ obtained using FBP-ChangAC and OSEMNoAC with headrest were higher than those without headrest. Images of middle brain region $(D+F)$ obtained using OSEMChangAC and OSEM-CTAC with headrest did not differ from those without headrest.

image reconstruction method is OSEM and ChangAC is used, the effect of the headrest should be taken into consideration.

At present, there is a high possibility that the presence of a headrest will affect reconstruction and AC of SPECT images for all methods other than OSEM-CTAC. Therefore, OSEM-CTAC must be considered the desired method. If other methods are used, the anterior brain region may have a higher count ratio value than the posterior region.

Since the headrest is generally made of carbon, which has a CT value of approximately 300 Hounsfield units, the headrest might cause $\gamma$-ray attenuation. A future possibility might be to construct the headrest from a material with a CT value close to that of air.
There are limitations to this study. First, SPECT acquisitions with no headrest might result in such issues as motion artifacts. Second, since we scanned only healthy volunteers, extrapolation to patients in actual clinical practice is limited.

\section{CONCLUSION}

For FBP-ChangAC, OSEM-ChangAC, and OSEM-NoAC, there were significant differences in $A+B / G$ ratio between images obtained with a headrest and those obtained without. In contrast, for OSEM-CTAC, there were no significant differences in $A+B / G$ ratio regardless of whether a headrest was used. Thus, the possible influence of a headrest on image reconstruction and $\mathrm{AC}$ need not be considered when OSEM-CTAC is used; other reconstruction methods may produce a higher $\mathrm{A}+\mathrm{B} / \mathrm{G}$ ratio.

\section{DISCLOSURE}

No potential conflict of interest relevant to this article was reported.

\section{REFERENCES}

1. Matsuda H, Mizumura S, Soma T, Takemura N. Conversion of brain SPECT images between different collimators and reconstruction processes for analysis using statistical parametric mapping. Nucl Med Commun. 2004;25:67-74.

2. Kanetaka H, Matsuda H, Asada T, et al. Effects of partial volume correction on discrimination between very early Alzheimer's dementia and controls using brain perfusion SPECT. Eur J Nucl Med Mol Imaging. 2004;31:975-980.

3. Johnson KA, Mueller S, Walshe T, English R, Holman BL. Cerebral perfusion imaging in Alzheimer's disease: use of single photon emission computed tomography and iofetamine hydrochloride I 123. Arch Neurol. 1987;44:165-168.

4. Cohen MB, Graham L, Lake R, et al. Diagnosis of Alzheimer's disease and multiple infarct dementia by tomographic imaging of iodine-123 IMP. J Nucl Med. 1986;27:769-774.

5. Imamura T, Ishii K, Sasaki M, et al. Regional cerebral glucose metabolism in dementia with Lewy bodies and Alzheimer's disease: a comparative study using positron emission tomography. Neurosci Lett. 1997;235:49-52.

6. Donnemiller E, Heilmann J, Wenning G, et al. Brain perfusion scintigraphy with ${ }^{99 \mathrm{~m}} \mathrm{Tc}$ HMPAO or ${ }^{99 m}$ Tc-ECD and ${ }^{123}$ I-beta-CIT single-photon emission tomography in dementia of the Alzheimer-type and diffuse Lewy body disease. Eur J Nucl Med. 1997;24:320-325.

7. Lee-Tzuu Chang. A method for attenuation correction in radionuclide computed tomography. IEEE Trans Nucl Sci. 1978;25:638-643.

8. Seo Y, Wong K, Sun M, Franc B, Hawkins R, Hasegawa B. Correction of photon attenuation and collimator response for a body-contouring SPECT/CT imaging system. J Nucl Med. 2005;46:868-877.

9. Licho R, Glick S, Xia W, Pan T, Penney B, King M. Attenuation compensation in ${ }^{99 m}$ Tc SPECT brain imaging: a comparison of the use of attenuation maps derived from transmission versus emission data in normal scans. J Nucl Med. 1999;40:456-463.

10. Stodilka RK, Kemp B, Prato F, Kertesz A, Kuhl D, Nicholson R. Scatter and attenuation correction for brain SPECT using attenuation distributions inferred from a head atlas. J Nucl Med. 2000;41:1569-1578.

11. Ishii K, Hanaoka K, Okada M, et al. Impact of CT attenuation correction by SPECT/CT in brain perfusion images. Ann Nucl Med. 2012;26:241-247.

12. Vallabhajosula S, Zimmerman R, Picard M, Stritzke P, Mena I, Hellman RS. Technetium-99m ECD: a new brain imaging agent: in vivo kinetics and biodistribution studies in normal human subjects. J Nucl Med. 1989;30:599-604.

13. Radon J. About the determination of functions by their integral values along certain manifolds [in German]. Ber Sachs Akad Wiss. 1917;69:262-277.

14. Hudson HM, Larkin R. Accelerated image reconstruction using ordered subsets of projection data. IEEE Trans Med Imaging. 1994;13:601-609.

15. Takeuchi R, Yonekura Y, Matsuda H, Konishi J. Usefulness of a three-dimensional stereotaxic ROI template on anatomically standardised ${ }^{99 \mathrm{~m}} \mathrm{Tc}-\mathrm{ECD}$ SPECT. Eur J Nucl Med Mol Imaging. 2002;29:331-341.

16. Farid K, Habert M, Martineau A, Caillat-Vigneron N, Sibon I. CT nonuniform attenuation and TEW scatter corrections in brain Tc-99m ECD SPECT. Clin Nucl Med. 2011;36:665-668. 